\title{
Contexto familiar y escolar de los alumnos absentistas de ESO: diferencias en padres y alumnos. Análisis en un centro educativo
}

\author{
Family and school context of the absentee pupils of Secondary School: \\ differences in parents and pupils. Analysis in an educational centre
}

\author{
María Carmen CABRERA HERRERA \\ Ayuntamiento de Cuenca \\ mccabrera@cuenca.es \\ María Elisa LARRAÑAgA RUBIO \\ Universidad de Castilla-La Mancha \\ elisa.larranaga@uclm.es
}

Recibido: $17 / 02 / 2014$

Revisado: $31 / 07 / 2014$

Aceptado: 20/10/2014

Disponible on line: 10/12/2014

\section{Resumen}

El absentismo escolar se presenta como un problema socio-educativo de naturaleza multicausal. Diversos estudios han analizado su naturaleza heterogénea a partir de los datos de los alumnos absentistas. Sin embargo, no se conocen estudios sobre absentismo donde se haya dado también participación a sus progenitores. Los objetivos de este estudio se dirigen a conocer las diferencias entre los contextos familiar y escolar de los alumnos absentistas y de los no absentistas en Educación Secundaria Obligatoria (ESO). El estudio se realizó en un Instituto que fue seleccionado por contar con el mayor número de alumnos absentistas en Cuenca. La muestra final está compuesta por 17 alumnos absentistas, 19 alumnos no absentistas y los progenitores de todos ellos. Se evaluó la comunicación y el clima familiar en los alumnos y en sus padres, la integración escolar y la participación en los procesos de acoso de los alumnos. Los resultados confirman que no existen diferencias entre ambos grupos en cuanto a la situación familiar que informan los padres y los alumnos. Por el contrario, sí que aparecen diferencias en cuanto a la situación escolar explicada por ambos grupos. Los alumnos absentistas indicaron que su integración escolar era menor en la dimensión de afecto, considerando que eran menos aceptados por los profesores. También los alumnos absentistas informaron que ejercían más conductas de acoso hacia sus compañeros.

Palabras clave: absentismo escolar, acoso escolar, integración escolar, clima familiar, comunicación familiar.

\begin{abstract}
Truancy is presented as a socio-educational problem of multiple causes. Wide studies have analyzed his heterogeneous nature based on the information of the absent pupils. However, there are not studies about absenteeism in which the progenitors of the pupils had been involved too. The objectives of this study aimed for knowing the differences between the family and school contexts of the absent and not absent pupils. The study was performed in a high school with more absent pupils compared to other educational centers. The final sample was composed of 17 absent pupils, 19 non absent pupils and their parents. The communication and the family climate between the pupils and their parents, the social integration and the participation in the process of harassment of the pupils were evaluated. The results confirm that there are no differences between the groups in terms of family situation that inform parents and students. By contrast, differences appear in the educational situation explained by both groups.

The absent students indicated that their school integration was lower in the dimension of affection, considering they were less accepted by teachers. Also absent students reported exercising more harassing behavior toward peers.

Keywords: school truancy, bullying, school membership, family climate, familiar communication.

Referencia normalizada: Cabrera Herrera, M. C. y Larrañaga Rubio, M. E. (2014): «Contexto familiar y escolar de los alumnos absentistas de ESO: diferencias en padres y alumnos. Análisis en un centro educativo». Cuadernos de Trabajo Social, 27(2): 385-394.
\end{abstract}

Sumario: Introducción. 1. Método. 2. Resultados. 3. Discusión. 4. Referencias bibliográficas. 


\section{Introducción}

El absentismo escolar se presenta como un grave problema socio-educativo cuyos protagonistas directos son aquellos alumnos que, encontrándose en periodo de escolaridad obligatoria (6 a 16 años), dejan de asistir de forma injustificada al centro educativo provocando, en muchos casos, el abandono definitivo del sistema escolar. El absentismo se manifiesta como una respuesta de rechazo por parte del alumno hacia el sistema escolar que puede adoptar varias formas: faltas de puntualidad, inasistencia a clase (en los extremos horarios), ausencias intermitentes (a clases o asignaturas), abandono esporádico del centro a determinadas horas, etc., hasta llegar al abandono definitivo (Uruñuela, 2005). Pero ¿qué puede llevar al alumno a rechazar el sistema escolar? En la búsqueda de respuestas a esta pregunta, García (2005), señala que en el estudio de las causas que favorecen la aparición del absentismo se debe tener en cuenta su naturaleza heterogénea, dinámica y cambiante, y que no es un fenómeno específico de determinados grupos sociales o étnicos.

El absentismo escolar ha sido un tema central en todas las políticas socio-educativas, que se evidencia en el desarrollo de programas específicos destinados a erradicarlo. En nuestra región, la Junta de Comunidades de Castilla-La Mancha desarrolló planes de acción conjunta en cuya intervención - la Orden 09/03/2007- se implicó a las Consejerías de Educación, Ciencia y Cultura y de Salud y Bienestar Social. Un año después, bajo el mismo marco normativo, ambas Consejerías establecieron el Protocolo Regional de Absentismo Escolar, que fue aprobado por la Comisión Regional para la Evaluación y Seguimiento del Absentismo Escolar, con fecha 29 de octubre de 2008. El Protocolo Regional de Absentismo Escolar de Castilla-La Mancha establece que, una vez detectada la situación de absentismo del menor y valoradas las causas que lo originan, el centro elaborará el correspondiente plan de intervención con el alumno. Si a lo largo de la intervención la situación no mejora o se observa que las causas van más allá de las centradas en el propio alumno y en el centro educativo, el caso será comunicado por el centro escolar a los Servicios Sociales Básicos del municipio. Su aplicación motivó que los Servicios Sociales Básicos del Ayuntamiento de Cuenca (Área de Intervención Social), al asumir la responsabilidad que dicha Orden les confería, realizara un análisis de los casos de absentismo recibidos de los centros educativos de la ciudad de Cuenca, con objeto de conocer qué factores socio-familiares (entorno familiar y social) podían estar relacionados con su aparición.

Los casos de absentismo analizados, correspondieron a los cursos 2007/2008, 2008/2009 y 2009/2010 (Comisión Local de Absentismo Escolar, 2010). Habían sido comunicados un total de 85 casos de Educación secundaria obligatoria (ESO), correspondientes a los siete centros de secundaria del municipio. Los datos recogidos confirmaron que el absentismo se daba con mayor intensidad en los alumnos de Secundaria (12 a 16 años) (Martínez-Arias, Aguado, Álvarez-Monteserin, Colodrón y Gallego, 2007). Al igual que en otras investigaciones (Broc, 2010; Martínez, 2011), los índices de absentismo variaban entre los centros.

Existe consenso en la comunidad científica sobre el papel que desempeña la familia como inhibidor y neutralizador de la implicación de los adolescentes en las conductas desviadas. Las relaciones que se dan entre los miembros de la familia representan el clima familiar y queda reflejado en el grado de comunicación, cohesión e interacción que existe entre los miembros (Zavala, 2001). Un clima familiar basado principalmente en el apoyo, el diálogo, el afecto y la comunicación entre sus miembros, constituye el factor principal para una elevada autoestima en todas sus dimensiones: física, emocional, familiar, académica y social (Musitu, Buelga, Lila, y Cava, 2001). Un clima familiar positivo puede prevenir la aparición de conductas desajustadas en los adolescentes (Megías, Elzo, Megías, Méndez, Navarro y Rodríguez, 2002) y, a su vez, amortiguar el malestar psicológico que sufren algunos adolescentes que son víctimas de violencia escolar (Povedano, Hendry, Ramos y Varela, 2011). Por el contrario, si los menores presencian conflictos de los padres envueltos de violencia verbal y física suponen un alto factor de riesgo que los predispone a la conducta violenta (Castañeda, Garrido-Fernández y Lanzarote, 2012). La observación de la violencia en el contexto familiar favorece que los niños y niñas aprendan a ejercer la violencia contra otras personas (Gámez-Guadix y Calvete, 2011). Sin embargo Rojas, Alemany y Ortiz (2011), en un estudio sobre el abandono escolar temprano, in- 
dicaron que sólo en un pequeño porcentaje de los alumnos que habían abandonado se daban circunstancias negativas en su casa.

Por otra parte, Gifford-Smith y Brownell (2003) han señalado que en el desarrollo de problemas de conducta durante la adolescencia también puede estar implicado un clima escolar negativo, caracterizado por un trato impersonal e indiferente del profesor hacia sus alumnos; condiciones que pueden ser generadoras de comportamientos agresivos y antisociales que interfieran en el clima escolar (Estévez, Martínez y Jiménez, 2009). Jiménez y Lehalle (2012) sostienen que la percepción de falta de afecto puede generar un malestar subjetivo en el alumno e influir en el desarrollo de conductas violentas hacia los iguales. Martínez, Moreno, Amador y Oxford (2011) también confirman este planteamiento, apuntando que una percepción o sentimiento negativo del alumno de su estancia en el aula (menor integración, menor valoración de los profesores) lleva implícitos comportamientos de transgresión de las normas de convivencia.

La violencia escolar, se define como aquella conducta intencionada, mediante la cual, se causa un daño a otra persona dentro del centro educativo o en cualquier actividad organizada por este, pudiendo ser ejercida o padecida por cualquier miembro de la comunidad educativa (Guerra, Álvarez-García, Dobarro, Núñez, Castro y Vargas, 2011). La aparición de estos problemas en los centros educativos de Educación secundaria preocupa cada vez más, pues afecta a la convivencia social, escolar, y al deterioro de las relaciones interpersonales (Morales y Trianes, 2012). García (2005), afirma que es la interacción de factores (acumulación de pequeñas rupturas en el ámbito personal, familiar o escolar), lo que desencadena las situaciones de riesgo que podrán contribuir a que los alumnos sigan, también, trayectorias absentistas. Para autores, como Bergeson y Heuschel (2003), Rué (2004) y González (2006) la ausencia de lazos sociales entre alumnos y profesores constituye un factor desencadenante de actitudes de rechazo a la escuela. Pérez-Serrano y Pérez de Guzmán (2011), informan que existe interrelación entre absentismo escolar y desarrollo de problemas de conducta en la escuela como la agresión escolar. Martínez (2011), encontró que los alumnos absentistas se relacionaban peor con los profesores y ejercían más actos de acoso a sus compañeros que los alumnos no absentistas. Sin embargo, otros autores han señalado que son algunas víctimas de acoso las que tienen un sentimiento de total rechazo al colegio que puede desencadenar en absentismo escolar (Varjas, Henrich y Meyers, 2009).

Es por ello por lo que el absentismo debe entenderse como un síntoma de algo que no funciona bien en el entorno del menor (Kronholz, 2011), un efecto llamada (Martín, Pizarroso y Sánchez, 2013), donde antes de intervenir será imprescindible identificar los factores de riesgo (Rué, 2004).

Corville-Smith, Ryan, Adams y Dalicandro (1998), realizaron un estudio sobre absentismo escolar para determinar la asociación entre la asistencia del alumno a clase y la influencia de los factores personales, familiares y escolares. La muestra estuvo constituida por 54 alumnos de educación secundaria (27 absentistas y 27 no absentistas). Los autores encontraron diferencias significativas entre ambos grupos, en aspectos como autoestima, disciplina y control parental, autoconcepto académico y competencia social en el aula.

Lozano (2003) planteó que una manera adecuada de profundizar en el conocimiento del absentismo es investigar en centros educativos concretos. Al realizar estudios centrados en institutos concretos se puede obtener un mayor conocimiento de las necesidades del alumnado absentista (Broc, 2010). Partiendo de esta premisa, nuestra unidad de análisis será el centro educativo para analizar los factores familiares y escolares que diferencian a los alumnos que asisten al mismo de forma regular de los que no lo hacen.

Nuestra investigación pretende contribuir al conocimiento del absentismo en Educación Secundaria Obligatoria en los contextos cercanos al alumno adolescente, concretamente la familia y la escuela. Aunque generalmente la conducta de la familia sólo queda recogida según como la perciben los hijos (Martínez, Aguado, Álvarez, Colondrón y Gallego, 2007), creemos que es fundamental también conocer la percepción global de la familia sobre su contexto. Por ello, para conocer la situación familiar que rodea al alumno, nos hemos centrado en la percepción sobre sus relaciones familiares y, también, cómo las perciben sus padres y madres (en lo sucesivo se utilizará el genérico padres para facilitar la 
lectura). En el contexto escolar analizaremos la integración en el centro y la violencia entre iguales que informan los alumnos. Los objetivos de la investigación se dirigieron a conocer las diferencias a nivel familiar y escolar entre alumnos absentistas y no absentistas y a determinar el peso del contexto familiar y escolar en el absentismo. Nos planteamos las siguientes hipótesis:

- H1: Existen diferencias entre la situación familiar (clima y comunicación familiar) informada por los padres y sus hijos absentistas y lo que informan los padres y sus hijos no absentistas.

- H2: Los alumnos absentistas presentan mayores problemas en el centro educativo en cuanto a integración y acoso que los no absentistas.

- H3: Las variables familiares y escolares inciden sobre el absentismo.

\section{Método}

\subsection{Participantes}

El estudio se realizó en un instituto de la ciudad de Cuenca que contaba con mayor número de alumnos absentistas comunicados a los Servicios Sociales básicos. El número total de participantes estuvo sujeto al número total de casos de absentismo que se daban en los cuatro cursos de Educación Secundaria Obligatoria del Instituto seleccionado para el estudio (I.E.S. Pedro Mercedes), durante el curso escolar 2011-2012. En función de los casos de absentismo existentes (20), se eligió, de forma aleatoria, el mismo número de casos de alumnos que asistían a clase de forma regular. Se constituyeron para la investigación dos grupos (absentistas y no absentistas), iguales en número y del mismo curso y aula. En cuanto a sexo, ambos grupos también estaban equiparados. Los casos de absentismo estaban distribuidos entre los cursos de primero (9 casos), segundo (10 casos) y tercero (1 caso). Esta incidencia del absentismo coincidía con lo informado por Martinez et al., la mayor parte de los absentistas comienzan a faltar a clase en el primer curso de ESO, coincidiendo con el cambio desde la educación primaria y continúan iniciándose en $2^{\circ}$ y $3^{\circ}$, siendo menos los que comienzan en el $4^{\circ}$ curso.

Con la colaboración de los Servicios Sociales básicos del Ayuntamiento de Cuenca (Área de Intervención Social), se pudo acceder a los alumnos ( 6 casos) que presentan absentismo severo o de alta intensidad (las faltas de asistencia a clase superan 50 por ciento del tiempo lectivo mensual). El acceso a este tipo de muestra es difícil, motivo por el que los alumnos con absentismo severo no suelen estar representados en otros estudios (idem, 2007). La muestra final la formaron 17 alumnos absentistas que aceptaron colaborar voluntariamente en la investigación y 19 alumnos no absentistas de primer a tercer curso de ESO y sus progenitores (participaron en total 36 familias: 20 cuestionarios fueron completados solamente por la madre, 3 por el padre y 13 por ambos). La no participación de alumnos de cuarto curso en la muestra obedeció a que no existía ningún caso de absentismo en este curso.

\subsection{Instrumentos}

Escala de clima social familiar (FES), de Moos y Moos (1981), versión española de FernándezBallesteros y Sierra (1989). Moos y Moos analizan el clima familiar como un concepto multidimensional de la interacción entre los miembros de la familia, donde el foco está en cómo funciona el sistema familiar y se mantiene como una unidad. La fiabilidad de las subescalas según el alfa de Cronbach fue $\alpha=0,82$ para la subescala de Cohesión, $\alpha=0,36$ para la subescala de Expresividad y $\alpha=0,77$ para la subescala de Conflicto.

Cuestionario de comunicación familiar (CA$M / C A-P)$, desarrollado por Barnes y Olson (1982) y adaptado en España por Musitu, Buelga, Lila y Cava (2001). Esta escala ya ha sido aplicada previamente en investigaciones sobre el ajuste escolar (Musitu, Estévez y Emler, 2007). Está compuesto por dos escalas tipo Likert: la primera escala evalúa la comunicación entre los hijos y la madre (desde el punto de vista de los hijos) y la segunda la comunicación entre los hijos y el padre (también desde el punto de vista de los hijos). La fiabilidad de las subescalas, alfa de Cronbach, fue $\alpha=0,88$ en Apertura en la comunicación con la madre, $\alpha=0,33$ en Problemas en la comunicación con la madre, $\alpha=0,92$ en Apertura en la comunicación con el padre y $\alpha=0,45$ en Problemas en la comunicación con el padre.

Cuestionario de comunicación familiar para padres (CFP), consiste en una adaptación del cuestionario de comunicación familiar de Musi- 
tu y otros. (2001) para su aplicación a los padres (Yubero, Larrañaga, Navarro y Ovejero, 2012). El cuestionario consta de 20 ítems similares a la escala de los hijos en los que se ha adaptado la redacción del enunciado para preguntar a los progenitores. La fiabilidad de las subescalas, según el alfa de Cronbach, fue $\alpha=0,70$ en Apertura comunicación con los padres y $\alpha=0,34$ en Problemas en la comunicación con los padres.

Percepción psicológica de integración esco$l a r$, se ha empleado la adaptación española realizada por Yubero, Ovejero y Larrañaga (2010) de la Psychological Sense of School Membership (PSSM, Goodenow, 1993), que mide la percepción de sentirse miembro del centro educativo. La fiabilidad obtenida según el alfa de Cronbach por factores fue $\alpha=0,83$ para Pertenencia, $\alpha=0,49$ para Rechazo y $\alpha=0,74$ para Afecto.

Cuestionario de acoso escolar (CAME), es un cuestionario para medir el acoso escolar entre iguales desde el punto de vista del agresor y de la víctima. Este cuestionario toma como referente el cuestionario desarrollado por Rigby y Bagshaw (2003), Instrument to asses the incident of involvelment in bully/victim interactions at school. Este instrumento ya ha sido aplicado anteriormente en diversas investigaciones sobre acoso entre adolescentes en España (Navarro, Yubero, Larrañaga y Martínez, 2012; Yubero, Ovejero y Larrañaga, 2010). La fiabilidad obtenida, alfa de Cronbach, fue $\alpha=0,57$ para la escala de Victimización y $\alpha=0,71$ para la escala de Agresión.

Los alumnos completaron la Escala de clima social familiar (FES), el Cuestionario de comunicación familiar (CA-M/CA-P), la Percepción psicológica de integración escolar (PSSM) y el Cuestionario de acoso escolar (CAME). Los padres contestaron el Cuestionario de comunicación familiar para padres (CFP) y a la Escala de clima social familiar (FES), indicando la persona que los completaba (padre, madre, ambos). En los adolescentes se ha recogido información sobre: edad, sexo, rendimiento académico y frecuencia de asistencia a clase.

\subsection{Procedimiento}

Se contactó con la dirección de los Servicios Sociales básicos del Ayuntamiento de Cuenca (Área de Intervención Social) para solicitar su colaboración y autorización para recabar la in- formación necesaria. Una vez concedida la autorización, se revisaron los casos de absentismo de Secundaria que habían sido comunicados por los distintos centros educativos al Área de Intervención Social, según el Protocolo Regional de Absentismo. De todos los centros revisados de la ciudad destacaban dos, por ser los que más casos de absentismo habían comunicado a los Servicios Sociales durante el curso 2011-2012 (coincidían con los resultados de los cursos 2007 a 2010). Se contactó telefónicamente con ambos y uno de ellos mostró su negativa a colaborar. El equipo directivo del otro centro seleccionado aceptó y mostró su interés en participar. El Departamento de Orientación del centro informó de todos los casos de absentismo, por curso y aula, con los que estaban interviniendo, incluidos los de absentismo severo, y de forma aleatoria seleccionó la muestra de alumnos no absentistas (en la misma proporción que no absentistas por curso y aula).

Para garantizar la participación de los alumnos absentistas y de sus padres, se contactó telefónicamente con estos para informarles de la investigación y pedirles su colaboración y la de sus hijos, proponiéndoles asistir a una reunión informativa individualizada (padre/madre o ambos), y en caso de que no pudieran asistir, se realizaría dicha reunión en el domicilio. En catorce casos asistieron a la reunión individualizada (en dos casos asistieron ambos progenitores, en los doce restantes fueron las madres), se les entregaron los respectivos sobres con los cuestionarios para que los cumplimentaran ellos y sus hijos. En tres casos la información y los cuestionarios se entregaron en los respectivos domicilios de los alumnos. Para la entrega de los cuestionarios cumplimentados se les dio la opción de hacerlo en el Departamento de Orientación del centro, en el Área de Intervención Social o bien que se pasara a recogerlos en el domicilio. Nueve sobres fueron devueltos cumplimentados al Área de Intervención Social, cuatro fueron entregados al Departamento de Orientación del centro, dos fueron recogidos en el lugar de trabajo de los respectivos padres de dos alumnos (manifestaron imposibilidad de llevarlo personalmente). Dos fueron recogidos en los respectivos domicilios y en otros tres domicilios los padres informaron que no los habían cumplimentado porque no querían participar ni ellos ni sus hijos (dos de ellos habían roto los cuestiona- 
rios). En total, los cuestionarios entregados de alumnos absentistas fueron 17 .

A los alumnos no absentistas se les informó telefónicamente y por carta a sus padres, solicitando también su colaboración y la de sus hijos. Los sobres con los cuestionarios fueron entregados a los alumnos por la educadora del centro en las horas de tutoría. De los cuestionarios entregados a los alumnos no absentistas 19 fueron devueltos cumplimentados al Departamento de Orientación.

Cada sobre entregado incluía dos cuadernillos, que contenían respectivamente, las cuatro escalas que debían cumplimentar los alumnos y las dos escalas que debían cumplimentar los padres. Los cuestionarios fueron identificados con un código para su posterior emparejamiento (hijo/padres).

\subsection{Análisis estadísticos}

Se utilizó el paquete estadístico SPSS 19.0 para Windows. Se realizó la prueba $t$ de Student para muestras independientes con el objeto de comparar las variables en los dos grupos (absentistas y no absentistas) respecto de la variable de estudio (absentismo). También se realizó prueba $t$ de Student para muestras relacionadas a fin de comparar el clima familiar y la comunicación informada por los hijos y las relaciones informadas por los padres en ambos grupos (absentistas y no absentistas). Con el objeto de confir- mar la implicación de las variables de estudio en el absentismo escolar se realizó una regresión logística binaria.

\section{Resultados}

Como se puede observar en la Tabla 1 , no existen diferencias significativas en ningún factor del clima familiar ni de la comunicación familiar entre la percepción que informaron los alumnos absentistas y los no absentistas, y los padres de ambas muestras. Padres e hijos informaron de características similares en cuanto al clima familiar.

Respecto a las variables escolares (Tabla 2), los alumnos no absentistas informaron de mayor percepción de afecto y aceptación por parte de los profesores. En la intervención en acoso los alumnos no absentistas informaron también que sufrían más acoso físico indirecto. Por el contrario la implicación en el rol de agresor es superior en los alumnos absentistas.

La integración escolar y las variables de acoso que resultaron significativas como predictoras del absentismo muestran un modelo ajustado $\left(\chi^{2}=32.74 ; \mathrm{gl}=6\right.$ y $\left.p<0,000\right)$. El coeficiente $R^{2}$ de Nagelkerke indica que un 82 por ciento de la variabilidad en la variable de respuesta viene explicada por el modelo de regresión logística. Asimismo, en los resultados de las clasificaciones de los casos según el valor observado y el valor pronosticado, se observa que el porcenta-

\begin{tabular}{|c|c|c|c|c|c|c|c|c|c|c|c|c|c|c|c|c|}
\hline & \multicolumn{6}{|c|}{ Alumnos } & \multicolumn{6}{|c|}{ Padres } & \multicolumn{4}{|c|}{ Hijo/Padres } \\
\hline & \multicolumn{2}{|c|}{$\begin{array}{c}\text { No } \\
\text { absentistas }\end{array}$} & \multicolumn{2}{|c|}{ Absentistas } & \multirow[t]{2}{*}{$t$} & \multirow[t]{2}{*}{$p$} & \multicolumn{2}{|c|}{$\begin{array}{c}\text { No } \\
\text { absentistas }\end{array}$} & \multicolumn{2}{|c|}{ Absentistas } & \multirow[t]{2}{*}{$t$} & \multirow[t]{2}{*}{$p$} & \multicolumn{2}{|c|}{$\begin{array}{c}\text { No } \\
\text { absentistas }\end{array}$} & \multicolumn{2}{|c|}{ Absentistas } \\
\hline & $M$ & $S x$ & $M$ & $S x$ & & & $M$ & $S x$ & $M$ & $S x$ & & & $t$ & $p$ & $t$ & $p$ \\
\hline \multicolumn{17}{|l|}{ Clima Familiar } \\
\hline Cohesión & 1,82 & 0,20 & 1,81 & 0,28 & 0,14 & 0,891 & 1,91 & 0,10 & 1,88 & 0,11 & 0,83 & 0,412 & $-2,45$ & 0,025 & $-1,57$ & 0,138 \\
\hline Expresividad & 1,71 & 0,14 & 1,63 & 0,21 & 1,29 & 0,206 & 1,68 & 0,18 & 1,71 & 0,14 & $-0,63$ & 0,532 & 0,52 & 0,610 & $-1,51$ & 0,151 \\
\hline Conflicto & 1,32 & 0,15 & 1,29 & 0,32 & 0,30 & 0,765 & 1,23 & 0,13 & 1,19 & 0,13 & 1,05 & 0,301 & 1,68 & 0,110 & 1,81 & 0,092 \\
\hline \multicolumn{7}{|c|}{ Comunicación Familiar } & & & & & & & & & & \\
\hline Abierta madre & 4,17 & 0,64 & 3,84 & 0,82 & 1,30 & 0,201 & 4,14 & 0,38 & 3,96 & 0,44 & 1,25 & 0,219 & 0,77 & 0,452 & $-1,39$ & 0,190 \\
\hline Abierta padre & 3,82 & 0,90 & 3,39 & 0,98 & 1,23 & 0,201 & & & & & & & $-0,76$ & 0,458 & $-3,03$ & 0,013 \\
\hline Problemas madre & 2,34 & 0,41 & 2,52 & 0,37 & $-1,33$ & 0,194 & 2,40 & 0,40 & 2,60 & 0,33 & $-1,61$ & 0,116 & $-1,44$ & 0,168 & $-0,08$ & 0,937 \\
\hline Problemas padre & 2,52 & 0,49 & 2,59 & 0,43 & $-0,42$ & 0,674 & & & & & & & 1,02 & 0,322 & 0,63 & 0,539 \\
\hline
\end{tabular}

Tabla 1. Comparación de medias variables. Contexto familiar. 


\begin{tabular}{|c|c|c|c|c|c|c|c|}
\hline & \multicolumn{2}{|c|}{$\begin{array}{c}\text { No } \\
\text { absentistas }\end{array}$} & \multicolumn{2}{|c|}{ Absentistsas } & \multirow{2}{*}{$t$} & \multirow{2}{*}{$p$} & \multirow{2}{*}{$D$} \\
\hline & $M$ & $S x$ & $M$ & $S x$ & & & \\
\hline \multicolumn{8}{|c|}{ Integración escolar } \\
\hline Pertenencia & 3,74 & 0,91 & 3,40 & 0,55 & 1,31 & 0,199 & 0,049 \\
\hline Rechazo & 4,07 & 0,95 & 3,72 & 0,60 & 1,26 & 0,215 & 0,048 \\
\hline Afecto & 4,11 & 0,88 & 3,35 & 0,73 & 2,79 & 0,008 & 0,186 \\
\hline \multicolumn{8}{|l|}{ Victimización } \\
\hline Físico directo & 0,11 & 0,32 & 0,63 & 0,24 & 0,49 & 0,627 & 0,007 \\
\hline Físico indirecto & 0,37 & 0,50 & 0,00 & 0,00 & 3,06 & 0,004 & 0,216 \\
\hline Verbal directo & 0,37 & 0,50 & 0,24 & 0,44 & 0,85 & 0,401 & 0,021 \\
\hline Verbal indirecto & 0,32 & 0,67 & 0,18 & 0,39 & 0,75 & 0,459 & 0,016 \\
\hline Exclusión & 0,00 & 0,00 & 0,12 & 0,34 & $-1,60$ & 0,119 & 0,072 \\
\hline \multicolumn{8}{|l|}{ Agresión } \\
\hline Físico directo & 0,00 & 0,00 & 0,59 & 0,62 & $-4,15$ & 0,000 & 0,337 \\
\hline Físico indirecto & 0,00 & 0,00 & 0,23 & 0,44 & $-2,35$ & 0,025 & 0,140 \\
\hline Verbal directo & 0,32 & 0,58 & 0,88 & 0,78 & $-2,48$ & 0,018 & 0,154 \\
\hline Verbal indirecto & 0,05 & 0,23 & 0,00 & 0,00 & 0,94 & 0,352 & 0,026 \\
\hline Exclusión & 0,00 & 0,00 & 0,41 & 0,62 & $-2,91$ & 0,006 & 0,199 \\
\hline
\end{tabular}

Tabla 2. Comparación de medias variables. Contexto escolar.

je total de coincidencias es del 88,2 por ciento, lo que implica que el modelo permite realizar un buen pronóstico del absentismo escolar.

\section{Discusión}

Nuestro objetivo era conocer las diferencias del contexto familiar y escolar entre alumnos absentistas y no absentistas de Educación Secundaria Obligatoria y el peso que estos contextos tienen sobre el absentismo. Para ello se analizó el contexto familiar a través del clima y la comunicación familiar, y el contexto escolar a través de la integración en el centro y la implicación en la violencia entre iguales.

Los resultados obtenidos no nos permiten confirmar nuestra primera hipótesis, no existen diferencias entre lo informado sobre el clima y la comunicación familiar de los alumnos absentistas y sus padres y los alumnos no absentistas y sus padres. En ambos grupos, los alumnos valo- raron positivo su contexto familiar coincidiendo con sus padres. Los resultados coinciden con los informados por Rojas-Ruiz, Alemany y Ortiz (2011) en el sentido de que no existen siempre circunstancias familiares negativas. No obstante, es importante destacar que en la comunicación abierta con la figura paterna informada por los alumnos, y la comunicación abierta informada por los padres, aparecen mayores diferencias en los alumnos absentistas que en los alumnos no absentistas. Estas diferencias en la comunicación, unido a la escasa participación de la figura paterna en los alumnos absentistas, podrían ser relevantes para el absentismo. Estudios previos han señalado la importancia que tiene el apoyo del padre como factor de protección de los problemas de conducta en los adolescentes (Jiménez, Musitu y Murgui, 2005; Musitu, Martínez y Murgui, 2006). Cava (2011) también informó de la importancia que la calidad de la comunicación 
con la figura paterna tiene en el acoso escolar.

Los alumnos absentistas presentan mayores problemas de integración y acoso (H2). Los alumnos absentistas perciben que su integración escolar es menor en la dimensión de afecto, considerando que son menos aceptados por los profesores. Respecto a las conductas de acoso, los resultados revelan que son los alumnos absentistas quienes ejercen más actos de acoso directo sobre sus iguales. Estos resultados confirman lo informado por Martínez (2011) al considerar que los alumnos absentistas se relacionan peor con sus profesores y ejercen mayor acoso a sus compañeros.

Los resultados obtenidos se pueden explicar desde la conjunción de diversos planteamientos:

- Relación de la falta de afecto con el desarrollo de conductas violentas. Este planteamiento coincide con lo informado por Estévez, Martínez y Jiménez (2009), Martínez et al., (2011) y Jiménez y Lehalle (2012), quienes sostienen que la percepción o el sentimiento negativo del alumno sobre su estancia en el aula lleva implícitos comportamientos de transgresión de las normas de convivencia y puede determinar la aparición de conductas violentas hacia los iguales.

- Relación de la falta de afecto con el desarrollo de conductas absentistas. Este planteamiento coincide con lo informado por Bergeson y Heuschel (2003), Rué (2004) y González (2006) quienes relacionaron la ausencia de lazos sociales entre alumnos y profesores con actitudes de rechazo a la escuela.

- Relación de las conductas violentas con el absentismo. Este planteamiento coincide con lo informado por Pérez-Serrano y Pérez de Guzmán (2011) quienes relacionaron absentismo escolar con problemas de conducta en la escuela como la agresión escolar.

El procedimiento de regresión logística binaria parece ser un instrumento idóneo para hacer una buena predicción del absentismo escolar como muestran los porcentajes de casos bien clasificados que hemos obtenido. El absentismo aparece relacionado con el clima escolar desde la integración escolar en la dimensión afecto y desde el acoso escolar en el papel de acosador del alumno absentista. Se confirma parcialmente la tercera hipótesis.

Podemos concluir que el absentismo aparece relacionado con el contexto escolar. Los alumnos absentistas se diferencian de los no absentistas en que se sienten menos integrados y aceptados por los profesores y en la mayor implicación en conductas de acoso hacia sus compañeros. Los resultados obtenidos pueden ser de utilidad para el diseño de planes de trabajo efectivos para la prevención del absentismo desde una perspectiva individual y contextual, en la misma línea que la propuesta por Jiménez y Lehalle (2012).

Una aportación importante de este trabajo es la participación de los padres en el estudio. También es necesario resaltar la colaboración obtenida de los alumnos con absentismo severo y sus familias. No obstante, en futuras investigaciones sería interesante poder contar con la participación de ambos progenitores para conocer el efecto diferencial en los factores implicados en el desarrollo de conductas absentistas, pues en este estudio, ha sido mayoritaria la participación de las madres de los alumnos absentistas. Esta investigación presenta la limitación que se deriva de los instrumentos utilizados, pues al tratarse de medidas de autoinforme pueden tener ciertos sesgos por el hecho de ser el propio sujeto el informador. Además, para una mayor profundización en la relación entre estas variables sería necesario realizar estudios longitudinales. Es también importante señalar que los resultados presentados en este trabajo deben interpretarse con cautela debido al tamaño reducido de la muestra dado que está desarrollado en un único centro educativo. Para próximas investigaciones sería interesante ampliar este estudio a otros centros educativos y a diferentes comunidades.

\section{Referencias bibliográficas}

Barnes, H. L. y Olson, D. H. (1982). Parent-adolescent Communication Scale. En D. H. Olson (ed.), Family inventories: Inventories used in a National Survey of Families across the family life cycle (pp. 33-48). St. Paul: Family Social Science, University of Minnesota Press.

Bergeson, T. y Heuschel, M. A. (2003). Helping students finish school. Why students drop out and how to help them graduate. Assessment and research. Washington: Office of Superintendent of Public Instruction. Recuperado de http://www.k12.wa.us/research/pubdocs/ dropoutreport2006.pdf 
Broc, M. A. (2010). Un estudio de meta-análisis sobre las causas del absentismo escolar y propuestas de prevención e intervención para la educación primaria y secundaria obligatoria. Recuperado de: http://www.doredin.mec.es/documentos/00220100000002.pdf.

Castañeda, A., Garrido-Fernández, M. y Lanzarote, M. D. (2012). Menores con conducta de maltrato hacia los progenitores: un estilo de personalidad y estilos de socialización. Revista de Psicología Social, 27, 157-167.

Cava, M. J. (2011). Familia, profesorado e iguales: claves para el apoyo a las víctimas de acoso escolar. Psychosocial Intervention, 20, 183-192. Recuperado de: http://www.copmadrid.org/webcopm/publicaciones/social/in2011v20n2a6.pdf.

Comisión Local de Absentismo Escolar. (2010). Informe de la evolución de los casos remitidos por los Centros Educativos al Área de Intervención Social desde el curso 2007 - 2008 al curso 2009 - 2010. Cuenca: Ayuntamiento de Cuenca. (Manuscrito inédito).

Corville-Smith, J. A., Ryan, B. R., Adams, G. y Dalicandro, T. (1998). Distinguishing absentee students from regular attenders: The combined influence of personal, family, and school factors. Journal of Youth and Adolescence, 27, 629-664. doi: 10.1023/A:1022887124634.

Estévez, E., Martínez, B. y Jiménez, T.I. (2009). Las relaciones sociales en la escuela: el problema del rechazo escolar. Psicología Educativa, 15, 5-12. Disponible en: http://www.uv.es/lisis/belen/psicolog-educt.pdf.

Fernández-Ballesteros, R. y Sierra, B. (1989). Escalas de Clima Social FES, WES, CIES y CES. Madrid: TEA.

Gámez-Guadix, M. y Calvete, E. (2012). Violencia filioparental y su asociación con la exposición a la violencia marital y la agresión de padres a hijos. Psicothema, 24, 277-283.

García, M. (2005). Dificultades en la aproximación a las dimensiones del absentismo: luces y sombras a partir de las voces del profesorado y de algunas tipologías institucionales. Aula Abierta, 86, 55-74. Recuperado de: http://dialnet.unirioja.es/servlet/articulo?codigo=2328689.

Gifford-Smith, M. E. y Brownell, C. A. (2003). Childhood peer relationships: Social acceptance, friendships, and social network. Journal of School Psychology, 41, 235-284. Recuperado de: http://dx.doi.org/10.1016/S0022-4405(03)00048-7.

González, M. T. (2006). Absentismo y abandono escolar: Una situación singular de la exclusión educativa. Revista Electrónica Iberoamericana sobre Calidad, Eficacia y Cambio en Educación, 4 (1). Disponible en: http://www.rinace.net/arts/vol4num1/art1.pdf.

Goodenow, C. (1993). The psychological sense of school membership among adolescents: Scale development and educational correlates. Journal of Psychology in the Schools, 30, 79-90. doi: 10.1002/1520-6807(199301).

Guerra, C., Alvarez-García, D., Dobarro, A., Núñez, J. C., Castro, L. y Vargas, J. (2011). Violencia escolar en estudiantes de Educación Secundaria de Valparaíso (Chile): Comparación con una muestra española. Revista Iberoamericana de Psicología y Salud, 2, 75-98. Disponible en: $\mathrm{http}: / /$ www.redalyc.org/pdf/2451/245116403004.pdf.

Jiménez, T. I., Musitu, G. y Murgui, S. (2005). Familia, apoyo social y conducta delictiva en la adolescencia: efectos directos y mediadores. Anuario de Psicología, 36, 181-195. Disponible en: http://www.raco.cat/index.php/AnuarioPsicologia/article/view/61813/76125.

Jiménez, T. I. y Lehalle, H. (2012). La violencia escolar entre iguales en alumnos populares y rechazados. Psychosocial Intervention, 21, 77-89. doi: 10.5093/in2012v21n1a5.

Kronholz, J. (2011). Truants: The challenges of keeping kids in school. Education Next, 11, 32-38. Lozano, A. (2003). Factores personales, familiares y académicos que afectan al fracaso escolar en la Educación Secundaria. Electronic Journal of Research in Educational Psychology, 1, 43-66. Disponible en: http://www.investigacion-psicopedagogica.org/revista/articulos/1/espannol/Art_1_4.pdf.

Martín, H., Pizarroso, M. y Sánchez, A. (2013). La familia centrada en el niño: impotencia y desesperanza. Cuadernos de Trabajo Social, 26, 285-293. doi: 10.5209/rev_CUTS.2013.v26.n2.42542.

Martinez, B., Moreno, D., Amador, L.V. y Orford, J. (2011). School victimization among adolescents. An analysis from an ecological perspective. Psychosocial Intervention, 20, 149-160. doi: 10.5093/in2011v20n2a3. 
Martínez, J. L. (2011). El absentismo escolar en la escolaridad obligatoria: etiología del problema y caracterización socio-educativa del alumno absentista. Toledo: Consejo Económico y Social.

Martínez-Arias, R., Aguado, P., Alvarez-Monteserin, M. A., Colodrón, F. y Gallego, E. (2007). Prevalencia del absentismo escolar en la educación secundaria obligatoria: relaciones con variables sociodemográficas y familiares. Psicología Educativa, 13, 35-63.

Megías, E., Elzo, J., Megías, I., Méndez, S., Navarro, F. J. y Rodríguez, E. (2002). Hijos y padres: comunicación y conflictos. Madrid: Fundación de Ayuda contra la Drogadicción (FAD).

Moos, R. H. y Moos, B. S. (1981). Family Environment Scale manual. Palo Alto, Ca.: Consulting Psychologist Press.

Morales, F. M. y Trianes, M. V. (2012). Análisis de valores y actitudes en temas morales en estudiantes de Educación Secundaria. Psicología Educativa, 18, 65-77.Recuperado de: http://www.copmadrid.org/webcopm/publicaciones/educativa/edFO2012v18n1a4.pdf.

Musitu, G., Buelga, S., Lila, M. y Cava, M. J. (2001). Familia y adolescencia. Madrid: Síntesis.

Musitu, G., Estévez, E., y Emler, N. P. (2007). Adjustment problems in the family and school contexts, attitude towards authority, and violent behavior at school in adolescence. Adolescence, 42, 779-794.

Musitu, G., Martínez, B. y Murgui, S. (2006). Conflicto marital, apoyo parental y ajuste escolar en adolescentes. Anuario de Psicología, 37, 247-258. Recuperado de: http://www.redalyc.org/pdf/ 970/97012834003.pdf.

Navarro, R., Yubero, S., Larrañaga, E. y Martínez, V. (2012). Children's cyberbullying victimization: Associations with social anxiety and social competence in a Spanish sample. Child Indicators Research, 5, 281-295. doi: 10.1007/s12187-011-9132-4.

Pérez-Serrano, M. G. y Pérez de Guzmán, M. V. (2011). Aprender a convivir: el conflicto como oportunidad de crecimiento. Madrid: Narcea.

Povedano, A., Hendry, L. B., Ramos, M. J. y Varela, R. (2011). Victimización escolar: clima familiar, autoestima y satisfacción con la vida desde una perspectiva de género. Intervención Psicosocial, 20, 5-12. doi: 10.5093/in2011v20n1a1.

Rigby, K. y Bagshaw, D. (2003). Prospects of adolescent students collaborating with teachers in addressing issues of bullying and conflict in schools. Educational Psychology, 23, 535-546. doi: $10.1080 / 0144341032000123787$.

Rojas-Ruiz, G., Alemany, I., y Ortiz, M. M. (2011). Influencia de los factores familiares en el abandono escolar temprano. Estudio de un contexto multicultural. Electronic Journal of Research in Educational Psychology, 9, 1377-1402. Disponible en: http://www.investigacion-psicopedagogica.org/revista/articulos/25/english/Art_25_624.pdf.

Rué, J. (2004). El absentismo escolar como reto para la calidad educativa. Madrid: MEC, Subdirección General de Información y Publicaciones.

Uruñuela, P. M. (2005). Absentismo escolar. I Jornades «Menors en edat escolar conflictes $i$ oportunitats». Recuperado de: http://weib.caib.es/Documentacio/jornades/jornades_menors/p_urunuela.pdf.

Varjas, K., Henrich, C. C. y Meyers, J. (2009). Urban middle school students perceptions of bullying, cyberbullying, and school safety. Journal of School Violence, 8, 159-176. doi: $10.1080 / 15388220802074165$.

Yubero, S., Larrañaga, E., Navarro, R. y Ovejero, A. (28-29 junio, 2012). Family communication and cyberbullying: A study with victims and their parents. Comunicación presentada en la International Conference on Cyberbullying, París.

Yubero, S., Ovejero, A. y Larrañaga, E. (2010). Apoyo social percibido en el contexto escolar y victimización entre iguales durante la adolescencia. Revista de Psicología Social, 25, 283-293.

Zavala, G. (2001). El clima familiar, su relación con los intereses vocacionales y los tipos caracterológicos de los alumnos del quinto año de secundaria de los colegios nacionales del distrito del Rimac. Lima: Facultad de Psicología UNMSM. 